・物种概念专题・

\title{
我国植物种级水平分类学研究刍议
}

\author{
杨亲二* \\ (中国科学院华南植物园, 中国科学院植物资源保护与可持续利用重点实验室, 广州 510650)
}

\begin{abstract}
摘要: 对洪德元先生最近在《生物多样性》 (2016年第24卷第3期)发表的《关于提高物种划分合理性的意见》一文 中的部分观点进行了进一步阐述。强调我国植物确实还存在大量种级水平的分类学问题有待解决, 我国植物分类 学研究在一些重要发展阶段(如系统阶段和物种生物学阶段)上存在明显缺失, 需要弥补。指出分类学发展到今天, 不宜再强调“经典分类学”和“实验分类学”之分, 应采用多学科手段解决分类学问题; 我国应加强植物分类专著水 平的研究工作, 注意培养年轻一辈植物分类学专著工作者; 在分类处理中应用居群概念和统计学方法时应特别谨 慎; 在系统植物学中接受物种概念的多元性是必要的, 但要向达到广义的生物学种概念努力, 不宜以有所谓的“归 并派”和“细分派”之分为借口而完全主观地划分物种。
\end{abstract}

关键词: 多学科手段; 物种划分; 种级水平分类学

\section{Comments on species-level taxonomy of plants in China}

Qiner Yang ${ }^{*}$

Key Laboratory of Plant Resources Conservation and Sustainable Utilization, South China Botanical Garden, Chinese Academy of Sciences, Guangzhou 510650

\begin{abstract}
Very recently, Professor Deyuan Hong, the editor-in-chief of the ongoing Flora of Pan-Himalaya, put forward a series of proposals (which may be conveniently termed Hong's Ten Points) on raising rationality in species delimitation, a hotly-debated question in systematic biology [Hong DY (2016) Opinion on raising rationality in species delimitation. Biodiversity Science, 24: 360-361]. The present paper is mainly to expound on some of the points. Even after the Flora Reipublicae Popularis Sinicae and its English version, Flora of China, was published, many species-level taxonomic problems of plants from China still remain to be solved. This situation may be due in part to previous unsatisfactory taxonomic practice and in part to our poor knowledge of taxonomic characters of a large number of plant groups from the country. Indeed, a very important stage in plant taxonomy, the biosystematic phase, was basically missing in China, and even the consolidation or systematic phase (one of the stages in alpha-taxonomy) has not yet been accomplished for some groups. Nowadays, it is inappropriate to emphasize the separation of orthodox and experimental taxonomy, and a multidisciplinary approach needs to be adopted in addressing taxonomic problems. To gain a fuller understanding of plant biodiversity in China, creative monographic work is badly needed, which requires the training of young botanical monographers. Moreover, it is suggested that practicing taxonomists should take extreme caution when employing population concept and statistical methods in their routine work. Finally, it is argued that every effort should be made toward a broader biological species concept although botanists have to accept the pluralism in species concept. To revert to a purely subjective species concept, under the pretext of the existence of the so-called lumpers or splitters in systematic biology, is not correct and must be opposed.
\end{abstract}

Key words: multidisciplinary approach; species delimitation; species-level taxonomy

最近，洪德元先生在《生物多样性》期刊发表 了《关于提高物种划分合理性的意见》一文(洪德元,
2016)。该文是针对目前他正在主持编研的Flora of Pan-Himalaya (《泛喜马拉雅植物志》) 而提出的十

收稿日期: 2016-08-17; 接受日期: 2016-09-06

基金项目: 国家自然科学基金(31370232)

* 通讯作者 Author for correspondence. E-mail: qeyang@scib.ac.cn 
点指导性意见(姑名之为“洪氏十条)”, 实际上也是 一位长期从事具体研究工作的分类学家(practicing taxonomist)对分类学核心内容——物种划分一一的 学术观点和实践经验的总结。所论虽极简短, 然均 切中肯綮, 我国年轻一辈植物分类学工作者实可视 其为对自己研究工作的“筬言”。我的分类学理论基 础和实际工作经验都十分有限, 但这篇论文也引起 了我对我国植物分类学尤其是种级水平分类学研 究中存在的一些突出问题及其可能的解决办法的 思考。

\section{1 我国植物还存在大量种级水平的分类学 问题有待解决}

《中国植物志》完成以后, 一般人都以为我国 植物的家底已基本摸清。但正如洪德元先生在其文 中指出的那样, 《中国植物志》是在客观条件、人才 培养和学术准备都不足的情况下启动和进行的, 其 中存在的有待解决的问题还很多。应当说稍后出版 的《中国植物志》英文修订版Flora of China解决了 其中一些问题, 但由于其中大部分类群的中方作者 还是原班人马, 很多外方作者对我国类群并不熟 悉, 所以不少类群其实只是中文版的翻译, 中文版 中存在的一些基本的种级水平分类问题在英文版 中仍然没有得到解决而遗留下来。试举菊科㯻吾属 (Ligularia Cass.)为例。该属的分类曾被认为已经比 较清楚(刘尚武等, 1994), 而我们和其他学者近年的 研究结果表明实际情况远非如此, 该属在《中国植 物志》和Flora of China中的处理都存在较多明显的 分类学问题(Ren \& Yang, 2010; 任琛和杨亲二, 2013a, b, c, 2014; Illarionova, 2013, 2014, 2016; Wang et al, 2016a, b)。其中大多数问题其实并未涉 及到复杂的性状变异式样分析, 解决起来相当简 单, 只要有关作者仔细研究原始文献和查看模式标 本(“尽可能查看模式标本” 是“洪氏十条”第八条), 就立即可以发现其中的错误并加以改正。这类比较 简单的基本分类学问题尚且同时出现在《中国植物 志》和Flora of China 中, 则涉及到较复杂的性状变 异式样的类群还存在较多问题就不值得奇怪了。可 以推想, 如果一个类群中有较多种类存在分类处理 问题, 则其检索表必然难以使用, 会给日常标本鉴 定带来诸多困难, 尤其对一些并非专门从事分类学 研究的相关领域学者的工作会(实际上已经)带来十
分不利的影响。要真正摸清我国植物的家底, 让植 物分类学家的工作成果能为其他领域和学科所用, 我国植物分类学家尤其是年轻一辈仍需继续努力。

\section{2 我国植物分类学研究在重要发展阶段上 存在明显缺失}

从植物分类学的整体发展历史来看, 一个地区 或类群的分类学研究大致可分为 4 个阶段: (1)先锋 或探索阶段(pioneer or exploratory phase), 这一阶段 的分类研究只基于少量标本; (2)巩固或系统阶段 (consolidation or systematic phase), 这一阶段的分类 研究基于较多标本和广泛的野外考察; (3)物种生物 学阶段(biosystematic phase), 这一阶段以前两个阶 段的工作为基础, 注重对类群的遗传学和细胞学研 究; (4)百科全书阶段(encyclopaedic phase), 这一阶 段的分类研究基于对各个不同学科的资料的综合 (Davis \& Heywood, 1963; Stace, 1980)。当然这些阶 段并非截然可分, 彼此存在重叠。前两个阶段也总 称为alpha-分类学(alpha-taxonomy), 特指基于外部 形态性状进行的分类。

欧、美和日本等少数地区和国家一方面由于植 物种类相对较少, 另一方面由于研究历史较长, 所 以其植物分类研究基本上达到了物种生物学阶段, 有些类群甚至达到了百科全书阶段。就我国的情况 而言, 至少种子植物的分类学研究应基本已完成先 锋或探索阶段, 完成巩固或系统阶段还有大量工作 要做, 物种生物学阶段的工作则基本缺失, 这导致 我国在植物一些重要性状方面的研究空白甚多, 并 进而导致很多分类学问题长期不能解决。本来, 在 《中国植物志》完成以后, 我国的植物分类学研究 理应继续完成系统阶段的研究, 同时进入物种生物 学阶段和百科全书阶段。但由于种种原因, 我国的 植物分类学研究迅速被边缘化, 从事这一学科的单 位和个人急骤减少。《中国植物志》完成之时又恰 逢分子系统学的兴起, 年轻一代工作者的兴趣迅速 转向DNA技术, 重心偏向于系统发育的研究。这些 状况不但使得我国只有少数类群进入了物种生物 学研究阶段, 而且很多类群的系统阶段的研究也没 有完成。这样, 与欧、美和日本等地区和国家相比, 我国的植物分类学研究缺失了几个极为重要的环 节, 导致我国物种水平的研究极为不足, 野外居群 观察十分不够, 对植物在居群水平上的变异缺少了 
解, 一些重要的物种生物学信息(目前这一术语已 较少使用。这里取其较广义的概念, 包括居群水平 上的宏观形态性状研究、微形态性状研究、细胞学、 细胞遗传学、繁育系统等方面)更是相当缺乏。例如 我国尚有约 800 属和 $93 \%$ 的种类缺乏染色体数目报道 (徐炳声等, 1996)。更为极端的情况是, 即使在毛茛 科广义升麻属(Actaea L. s.1.)这样具有重要药用价值 而种类又不多的类群，一般的外部形态性状信息都 还有很多空白。如南川升麻(A. nanchuanensis (P. K. Hsiao) J. P. Luo, Q. Yuan \& Q. E. Yang)描述50年后还 没有果实形态性状的报道(Luo et al, 2016a), 而短果 升麻(A. brachycarpa (P. K. Hsiao) J. Compton)描述50 年后还没有花部形态性状的报道(Luo et al, 2016b), 导致《中国植物志》和Flora of China 中对这两种植 物及该属其他种类的分类处理和地理分布记载都或 多或少存在一定的错误。确实, 要正确划分物种, “染色体、花粉以及其他学科提供的数据和资料都 可能是很有价值的, 甚至是不可或缺的。分类研究 必须采用综合手段” (洪氏第五条)。Jeffrey (1982)也 曾指出, 我们实际进行分类的东西是我们关于植物 的知识。换言之, 我们对植物各方面的性状知道得 越多, 则我们做出的分类才有可能更好。显然, 如 果不尽快促进我国植物分类学缺失阶段的完成以 弥补我国在植物重要分类性状方面的空白, 则要真 正解决我国植物种级水平的分类学问题从而切实 摸清我国植物的家底是不可能的。我国一些植物分 类学家对学科的理解存在误区, 学术思想和研究手 段都相对保守, 不注意利用其他学科的证据。记得 我国著名植物胚胎学家王伏雄先生晚年在一次研 究生答辩会上曾愤愤不平地提到, 他早期开展我国 植物花粉研究时需要从标本上采集少量材料, 但一 些分类学家无视他对这种研究与分类学有密切关 系的解释, 以对标本有损坏为由而加以阻挠。

值得指出的是, 上述对南川升麻分类处理和地 理分布记载的错误也直接导致该种曾被误认为是 仅分布于其模式产地的濒危植物, 并被选为我国濒 危植物的 8 个代表物种之一, 一些学者从不同方面 对其进行了濒危原因的研究(奇文清等, 1997, 1998; 符近等, 1998; 岳春雷和刘亚群, 1999; 岳春雷等, 2002)。现在看来, 这些研究的前提业已存在问题, 则其研究的意义和结论的正确性也就大打折扣。确 实, “合理的物种划分是生物多样性研究、保护和可
持续利用的基础” (洪氏第十条)。

\section{3 应跳出分类学中的“经典”框框}

所谓经典分类学(orthodox or classical taxonomy)或传统分类学(traditional taxonomy)实际上就是 上述alpha-水平的分类学, 主要基于外部形态性状 进行分类处理。这是分类学研究中不可缺少的一 步。但植物分类学发展到今天, 我们在实际研究工 作和分类学人才培养中似应努力跳出“经典”框框。 植物分类学是生物学中最基础的学科之一, 既具有 传统性, 又具有前沿性。由于这一学科本身没有自 己的资料, 主要依靠来自其他多个学科的证据解决 问题, 因此具有无穷的综合性(unending synthesis), 能不断吸收和利用不同学科的新证据改进以前的 研究结果, 随着其他学科的发展而不断进步。植物 分类学无穷综合性的特点决定了一位合格的植物 分类学家必须具备尽可能广泛的生物学背景(洪氏 第一条)。过去长期认为从事植物分类学研究只需具 备植物形态学、命名法规、拉丁文等方面的基本知 识的时代应当说早已过去。目前如果再对分类学中 的“经典”方面加以强调实际上已对学科的发展有害 无益。这一方面会让其他学科的学者轻视分类学家, 以为分类学研究易于进行, 另一方面也会使一些分 类学家固步自封, 有意无意将自己封为“经典”分类 学家, 不主动吸收或自己动手进行实验室工作以获 取其他学科的证据, 满足于泛泛认识一些植物而不 去努力解决真正的分类学问题(尽可能普遍认识植 物值得提倡, 但不宜满足于此, 毕竟科学研究应当 产生新的知识而不只是利用已有知识)。分类学发展 到今天, 分类学就是分类学, 不宜再强调所谓“经 典分类学”和“实验分类学(experimental taxonomy)” 之分; 分类学研究的无穷综合性决定了分类学家必 须随时注意其他学科的发展, 即“吸纳新思想、新方 法、新数据” (洪氏第四条)。

国家自然科学基金委员会2013年设立了“经典 植物分类学人才培养”重大资助项目, 中国科学院 有关机构长期以来也设有类似项目，用意是良好 的, 针对性也很强。但如将项目名称改为“植物分类 学专著水平研究人才培养” (Training Programme for Talents in Botanical Monography)则似更能反映出植 物分类学学科本身无穷综合性的特点和世界植物 分类学目前的发展以及人才培养现状。植物分类专 
著工作(botanical monography)是理解生物多样性的 基础, 价值难以估量, 也能为生物多样性保护和高 质量植物志书的编研提供重要的基础资料。由于分 类学专著要求包含所涉及类群尽可能全面的信息, 所以在培养年轻一辈分类学家(主要是博士研究生) 时, 如能为其选择一个大小适当的类群开展专著水 平的研究, 则有可能使其在攻读学位期间从形态到 分子证据的获取和分析方面都得到全面训练而成 为一名真正合格的分类学家。2011年国际植物分类 学协会主持出版的Monographic Plant Systematics:

Fundamental Assessment of Plant Biodiversity (Stuessy \& Lack, 2011) 是一本值得我国植物分类学 家特别是年轻一辈工作者关注的著作。该书首先指 出自分子系统学兴起以来, 年轻一代工作者的兴趣 迅速转向激动人心的DNA技术, 结果目前这一代工 作者失去了对植物分类专著工作的理解、经验甚至 兴趣, 这种状况现在必须改变。以后各章分别阐述 了植物分类专著工作在生物多样性保护中的重要 性及其涉及的各种性状数据(从形态到分子)、DNA 条形码、野外考察、分类学文献、植物绘图、植物 命名法规、植物学拉丁文、未来专著编研方式展望 诸方面, 深刻说明了从事真正意义的创新性植物分 类学研究需要具备极为广博的知识背景, 培养一位 合格的专著工作者 (monographer) 确实需要相当长 的时间。如能仔细通读该书, 对“洪氏十条”当更具 会心。该书还强调: 不能简单地以论文数量及其影 响因子来评价分类学专著工作, 因为这种工作经费 偏少, 实际上却十分费时费力, 一部专著的完成可 能需要花费 5-10年。分类学专著的影响因子不可能 很高, 但其价值可能大于 10 篇短的研究论文。过去 30 年分类学专著明显减少的原因之一也许是太过 于强调系统发育方法和分子方法了。系统发育方面 的研究无疑十分重要和有趣, 但由于有关资助和绩 效评价机构对其极度重视, 以致作为系统发育研究 基础的分类学专著工作遭到忽略, 得不到必要的激 励和经费支持, 这种状况对全面理解生物多样性及 其有效保护都十分不利。应当说这些意见确实是切 中时弊的。

\section{4 实际操作中应用居群概念和统计学方法 划分物种宜注意的问题}

简单而言, 居群概念的实质就是居群内的个体
皆有所不同，因而有差异的个体不一定就属于不同 的种。这一点说起来容易, 但在实际工作中其实是 存在陷阱的。在标本室和野外观察中, 在对一个类 群还没有深入认识的情况下, 分类学家很可能将生 长于某一具体地点的形态相似而实际上属于不同 物种的个体错误地认为属于一个物种同一居群内 的植物, 从而将种间差异视为居群内差异并进而作 出错误的分类处理。另一方面，对于较广布而存在 居群间变异的种类而言, 确定居群间的差异究竟属 于种内变异还是不同物种之间的差异有时也极其 困难。这两方面都可能导致分类处理错误。我在处 理毛莨科乌头属 (Aconitum L.) 的狭盔高头 $(A$. an gustius (W. T. Wang) W. T. Wang)和赣皖乌头 $(A$. finetianum Hand.-Mazz.) 时就差点犯这样的错误。这 两种植物在我国安徽黄山、湖南衡山、江西庐山同 域分布，在二者的模式产地江西庐山生长在一起， 在花梗毛被、花构造等乌头属相当重要的形态性状 方面没有区别, 我从标本室也看到同号标本有时被 鉴定为这两种植物, 所以最初判断它们可能只是代 表同一物种的居群内变异。后来经过仔细的野外观 察和广泛的细胞学研究, 发现二者的染色体倍性 (狭盔高乌头为四倍体, 而赣皖乌头为二倍体)和核 型组成明显不同, 同时发现以前将狭盔高乌头处理 为高乌头 (A. sinomontanum Nakai) (二倍体)的变种 (即var. angustius W. T. Wang) 也是不合适的, 以前被 鉴定为高乌头的很多居群实际上属于狭盔高乌头。 这就是确定居群间差异究竟属于种内变异还是不 同物种之间的差异有时也颇困难的情况(Gao et al, 2012; Hong et al, 2016)。毛莨科升麻属不同种类的 植物如短果升麻和单穗升麻(Actaea simplex (DC.) Wormsk. ex Prantl)在前者的模式产地云南东川也生 长在同一群落中, 二者形态较为相似, 采自该群落 的这两种植物的标本被编为同号并被鉴定为同一 种(Luo et al, 2016b)。采集人和鉴定人显然都有居群 概念, 但不幸都掉入陷阱中。Compton等(1998)将披 针叶升麻(Cimicifuga lancifoliolata X. F. Pu \& M. R. $\mathrm{Jia}=$ Actaea lancifoliolata (X. F. Pu \& M. R. Jia) J. P. Luo, Q. Yuan \& Q. E. Yang)错误处理为短果升麻的 异名也应该属于类似的情况。在前者的模式产地四 川大邑西冷雪山, 短果升麻也有分布; 披针叶升麻 的同举模式(paratype)标本(蒲旭峰89022 (PE))与采 自西冷雪山的一号短果升麻标本 (蒲旭峰 89021 
(PE))的采集号又正好相连。这显然误导了Compton 等(1998)的分类处理。实际上披针叶升麻是升麻属 中一种十分独特的植物, 与短果升麻易于区别(Luo et al, 2016b)。在分类学研究中, 强调“树立居群概 念、摒弃模式概念” (洪氏第三条)无疑极为重要, 但 具体操作过程中应特别小心, 千万不能以为形态相 似且生长在一起的个体就一定属于一个居群, 而形 态有所不同且又生长在不同地点的个体就一定属 于不同物种。我感到从事分类学研究确实需要长期 实际工作的历练, 需要不断积累处理不同具体问题 的经验, 深入的野外居群观察和尽可能利用多学科 手段开展研究尤其重要。

在分类学中利用统计学方法(主要是多元形态 分析(multivariate morphometrics)) 无疑也是十分重 要的(洪氏第三条), 但要特别注意在对所涉及植物 的性状有较清楚了解的情况下进行统计分析才有 望取得良好效果。如果在对一个类群的有关性状还 缺乏基本了解的情况下就率尔操触, 实际上并不能 真正解决问题。Lee和Park (1994)曾对升麻属亚洲一 些种类进行了统计分析, 但他们当时对其中一些植 物的重要性状如短果升麻的花部性状和南川升麻 的果实性状缺乏了解, 所以得出的结果与Compton 和Hedderson (1997)的统计分析结果存在明显不同, 也没有得到后来的形态学、细胞学、分子系统学等 研究结果(Compton et al, 1998; Ren et al, 2012; Luo et al, 2016a, b)的支持。因此, 首先应针对植物性状 本身进行充分研究, 然后再采用适当的统计学方法 进行分析。缺乏数学基础布庸讳言是生物学工作者 的软肋, 分类学家在进行统计学分析时最好能请教 有关统计学专家, 以保证统计分析过程及对其结果 解释的正确性。

\section{5 关于植物物种概念的多元性，兼论“归并 派”和“细分派”}

物种的概念和定义长期以来众说纷纭, 所以物 种的确定是分类学家最重要但同时又是最感困难 的一项工作。“生物学种(biological species)”概念是 目前最为广泛接受的物种概念, 但由于高等植物普 遍存在种间杂交、单亲本生殖和无融合生殖现象, 物种形成方式多样, 因此完全严格按照生殖隔离标 准来划分物种的“生物学种”概念在高等植物中是不 实际的。比较现实的选择是接受多元性的物种概念 (pluralistic species concept)或物种概念的多元性 (pluralism in species concepts) (Stebbins, 1993; Stuessy, 2009)。尽管如此, 对于大多数有性生殖植 物而言, Stebbins (1993)主张的广义的生物学种概念 (a broader biological species concept) (一方面强调生 殖隔离，同时也考虑到形态、遗传、生态等方面因 素)还是可取的, 至少是分类学家应当努力达到而 且通过深入的工作可以达到的目标。在实际工作中, 我们可先应用表征分类方法即根据性状的全面相 似性来划分类群(phenetic grouping), 以此为出发点 进行深入研究, 最后综合考虑各种因素而达到合理 划分种类的目标。这种工作当然是费时费力的。

由于系统生物学(systematic biology)中缺乏统 一的物种概念和物种划分标准, 加之长期以来有所 谓的“归并派(lumpers)”和“细分派(splitters)”之说(洪 氏第九条), 因此一些学者在物种划分上显得相当 随意, 甚至有意识地将自己归于细分派, 认为自己 的做法并不孤立，从而发表很多自然界实际上并不 存在的新物种。但实际上真正意义的细分派(如前苏 联柯马洛夫学派(Komarovian school))划分物种也是 有一定原则的。有些广布种确实存在多型性, 种下 可以划分出亚种、变种或变型。如果这些种下类型 确实属于有一定独立性的分类学实体(taxonomic entity), 有些学者认为这些实体应当处理为独立的 物种而不是种下类群, 这样的学者或可称为细分 派。但如果完全不考虑变异的间断性和性状的相关 性，仅基于少数标本甚至一份残破不全的标本从而 很难据以看出性状变异式样的情况下就随意描述 新种, 以致将采自一个具体产地的已经描述的物种 的标本反复描述为新种, 这样的作者就不能归于 “细分派”而只能称为“乱分派”了。植物学家固然不 必完全基于生殖隔离的有无(即严格的生物学种概 念)划分物种, 但也不能因此而完全主观地划分物 种。由于分类学工作的复杂性, 分类学家在工作中 出现一些错误是很难避免的, 但如果以分类学中有 “细分派”和“归并派”之说作为借口而随意发表新种 则是不可取的。这样的做法对学科的进步没有丝毫 贡献，反而给后人的工作增加许多负担。“乱分派” 的研究结果也会给其他分类学家带来不良的心理 效应(psychological effects): 如果一个类群被过分细 分, 那么后来的作者如果对这个类群不熟悉, 就很 容易将与这些已经被过分细分的种类不一致的植 
物描述为新种，最后导致无法收拾的局面(Davis \& Heywood, 1963)。

分类学家难免会受到发表新分类群所带来的 成就感和满足感的心理驱动, 同时一些刊物也有意 无意地助长了一些作者热衷于发表新种的行为。一 些刊物乐于接受发表描述新分类群的论文, 但不愿 意甚至拒绝发表将分类群归并为异名的论文。实际 上后一方面的工作也同样重要(Davis \& Heywood, 1963; 徐炳声, 1998)。当然轻易发表和轻易归并类 群都是不合适的，任何分类处理都应基于深入的 研究。

致谢: 感谢中国科学院植物研究所马克平研究员对 本文的细致修改。

\section{参考文献}

Compton JA, Hedderson TAJ (1997) A morphometric analysis of the Cimicifuga foetida L. complex (Ranunculaceae). Botanical Journal of the Linnean Society, 123, 1-23.

Compton JA, Culham A, Jury SL (1998) Reclassification of Actaea to include Cimicifuga and Souliea (Ranunculaceae): phylogeny inferred from morphology, nrDNA ITS, and cpDNA trnL-F sequence variation. Taxon, 47, 593-634.

Davis PH, Heywood VH (1963) Principles of Angiosperm Taxonomy. Oliver \& Boyd, Edinburgh \& London.

Fu J, Qi WQ, Gu ZH, You RL (1998) Seed dormancy and germination in Cimicifuga nanchuanensis. Acta Botanica Sinica, 40, 303-308. (in Chinese with English abstract) [符近, 奇文清, 顾增辉, 尤瑞麟 (1998) 南川升麻种子休眠与萌 发的研究. 植物学报, 40, 303-308.]

Gao Q, Ren C, Yang QE (2012) Taxonomic status and distributional range of Aconitum angustius (Ranunculaceae) based on cytological evidence. Nordic Journal of Botany, 30, 426-438.

Hong DY (2016) Opinion of raising rationality in species delimitation. Biodiversity Science, 24, 360-361. (in Chinese) [洪德元 (2016) 关于提高物种划分合理性的意见. 生物 多样性, 24, 360-361.]

Hong Y, Gao Q, Luo Y, Luo JP, Zhang Y, Yuan Q, Yang QE (2016) Karyology of Aconitum subgenus Lycoctonum (Ranunculaceae) from China, with a report of the new base chromosome number $x=6$ in the genus Aconitum. Nordic Journal of Botany, 34, 442-454.

Hsu PS (1998) The species problem in plant taxonomy in China. Acta Phytotaxonomica Sinica, 36, 470-480. (in Chinese with English abstract) [徐炳声 (1998) 中国植物分类学中 的物种问题. 植物分类学报, 36, 470-480.]

Hsu PS, Zhang ZY, Chen JK, Hong DY (1996) Advances in chromosome studies and plant taxonomy. Journal of Wuhan
Botanical Research, 14, 177-187. (in Chinese with English abstract) [徐炳声, 张芝玉, 陈家宽, 洪德元 (1996) 染色 体研究的进展与植物分类学(上). 武汉植物学研究, 14 , 177-187.]

Illarionova I (2013) Typification and specific status of Senecio lagotis W. W. Sm. (Asteraceae). Taiwania, 58, 221-225.

Illarionova I (2014) Taxonomic notes on sections Corymbosae and Subracemosae of genus Ligularia (Asteraceae). The Journal of Japanese Botany, 89, 365-375.

Illarionova I (2016) The identity of Ligularia euryphylla (Asteraceae, Senecioneae). Willdenowia, 46, 99-104.

Jeffrey C (1982) An Introduction to Plant Taxonomy. Cambridge University Press, London.

Lee HW, Park CW (1994) A systematic study of the Cimicifuga foetida L. complex and related species (Ranunculaceae). Journal of Plant Biology, 37, 111-124. (in Korean with English Abstract)

Liu SW, Deng DS, Liu JQ (1994) The origin, evolution and distribution of Ligularia Cass. (Compositae). Acta Phytotaxonomica Sinica, 32, 514-524. (in Chinese with English abstract) [刘尚武, 邓德山, 刘建全 (1994) 㯻吾属的起 源、演化与地理分布. 植物分类学报, 32, 514-524.]

Luo JP, Wang L, Ren C, Yang QE, Yuan Q (2016a) Taxonomic notes on Cimicifuga nanchuanensis (Ranunculaceae), a hitherto imperfectly known species from China. Nordic Journal of Botany, 34, 87-101.

Luo JP, Hong Y, Ren C, Yang QE, Yuan Q (2016b) Reinstatement of the Chinese species Cimicifuga lancifoliolata (Ranunculaceae). Nordic Journal of Botany, 34, doi: 10.1111/njb.01092.

Qi WQ, Feng Y, Chenzhu XZ, Li ZL, You RL (1997) Studies on the reproductive characteristics of Cimicifuga nanchuanensis (Ranunculaceae), an endemic endangered species to China. Acta Botanica Sinica, 39, 7-10. (in Chinese with English abstract) [奇文清, 冯云, 陈朱希昭, 李正理, 尤瑞麟 (1997) 濒危植物南川升麻生殖特性的研究. 植 物学报, 39, 7-10.]

Qi WQ, You RL, Chen XL (1998) Pollination biology in Cimicifuga nanchuanensis, an endangered species (Ranunculaceae). Acta Botanica Sinica, 40, 688-694. (in Chinese with English abstract) [奇文清, 尤瑞麟, 陈晓麟 (1998) 濒危植物南川升麻传粉生物学的研究. 植物学报, 40, 688-694.]

Ren C, Yang QE (2010) The identity of Ligularia yui (Asteraceae: Senecioneae) from China. Nordic Journal of Botany, 28, 716-719.

Ren C, Yuan Q, Yang QE (2012) Cytological evidence supports the taxonomic status of the Sino-Himalayan species Cimicifuga frigida (Ranunculaceae). Nordic Journal of Botany, 30, 585-595.

Ren C, Yang QE (2013a) Taxonomic studies on the genus Ligularia Cass. (Asteraceae, Senecioneae) from China (I): the identity of $L$. angustiligulata. Journal of Tropical and Subtropical Botany, 21, 351-355. (in English with Chinese ab- 
stract) [任琛，杨亲二 (2013a) 中国夢吾属(菊科-千里光 族)的分类学研究(一): 狭舌㯻吾的名实订正. 热带亚热 带植物学报, 21, 351-355.]

Ren C, Yang QE (2013b) Taxonomic studies on the genus Ligularia Cass. (Asteraceae, Senecioneae) from China (II): the identity of L. fangiana. Journal of Tropical and Subtropical Botany, 21, 389-396. (in English with Chinese abstract) [任 琛, 杨亲二 (2013b) 中国䝴吾属(菊科-千里光族)的分类 学研究(二): 植夫㯻吾的名实订正. 热带亚热带植物学 报, 21, 389-396]

Ren C, Yang QE (2013c) Taxonomic studies on the genus Ligularia Cass. (Asteraceae, Senecioneae) from China (III): the identities of L. brassicoides, L. ianthochaeta, and L. paradoxa var. palmatifida. Journal of Tropical and Subtropical Botany, 21, 538-542. (in English with Chinese abstract) [任 琛, 杨亲二 (2013c) 中国橐吾属(菊科-千里光族)的分类 学研究(三): 芥形㯻吾、岷县㯻吾和半裂䝴吾的名实订正. 热带亚热带植物学报, 21, 538-542.]

Ren C, Yang QE (2014) Taxonomic studies on the genus Ligularia Cass. (Asteraceae, Senecioneae) from China (IV): the identities of L. kangtingensis and Senecio ficariifolius. Journal of Tropical and Subtropical Botany, 22, 19-25. (in English with Chinese abstract) [任琛, 杨亲二 (2014) 中国 㯻吾属(菊科-千里光族)的分类学研究 (四): 康定㯻吾和 Senecio ficariifolius 的名实订正. 热带亚热带植物学报, 22, 19-25.]

Stace CA (1980) Plant Taxonomy and Biosystematics. Edward Arnold, London.

Stebbins GL (1993) Concepts of species and genera. In: Flora of North America (ed. Flora of North America Editorial Committee), pp. 229-246. Oxford University Press, New York \& Oxford.

Stuessy TF (2009) Plant Taxonomy: the Systematic Evaluation of Comparative Data, 2nd edn. Columbia University Press, New York.

Stuessy TF, Lack HW (2011) Monographic Plant Systematics: Fundamental Assessment of Plant Biodiversity. A. R. G. Gantner Verlag K. G., Ruggell.

Wang L, Luo HY, Ren C, Yang QE (2016a) Taxonomic and nomenclatural notes on Ligularia ghatsukupa, L. leesicotal and L. rumicifolia (Asteraceae, Senecioneae). Phytotaxa, 243, 260-270.

Wang L, Luo HY, Ren C, Yang QE (2016b) Cremanthodium botryocephalum and C. spathulifolium are both merged with Ligularia lagotis (Asteraceae, Senecioneae). Phytotaxa, 247, 274-280.

Yue CL, Liu YQ (1999) Preliminary studies on physiological ecology of photosynthesis of an endangered plant-Cimicifuga nanchuanensis. Acta Phytoecologica Sinica, 23, 71-75. (in Chinese with English abstract) [岳春 雷, 刘亚群 (1999) 濒危植物南川升麻光合生理生态的 初步研究. 植物生态学报, 23, 71-75.]

Yue CL, Jiang H, Zhu YM (2002) Analysis on numeric dynamics of population of Cimicifuga nanchuanensis, an endangered plant. Acta Ecologica Sinica, 22, 793-796. (in Chinese with English abstract) [岳春雷, 江洪, 朱荫湄 (2002) 濒危植物南川升麻种群数量动态的分析. 生态学 报, 22, 793-796.] 